\title{
AN EXTREMAL HARMONIC FUNCTION ${ }^{1}$
}

\section{KENNETH M. LARSEN}

Let $\Omega$ be a regular subregion of a locally Euclidean $n$-space $V$ with $\Omega$ having a finite number of border components partitioned into two disjoint sets $\alpha$ and $\beta$ (e.g. a spherical shell). Let $h$ be the harmonic function which vanishes on $\alpha$ and has the constant value $k$ on $\beta$ such that $\int_{\alpha}(\partial h / \partial n) d S=1$. The class of regular harmonic functions on $\Omega$ which are continuous on $\partial \Omega$, which vanish on $\alpha$ and which have been normalized so that $\int_{\alpha}|\partial u / \partial n| d S=1$ will be denoted by $H_{0}(\Omega)$. For $u \in H_{0}(\Omega)$, the harmonic mean $m(u ; k)$ is defined by $m^{2}(u ; k)$ $=\int_{\beta} u^{2}(\partial h / \partial n) d S$. We shall use $m(u ; \lambda)$ for the mean over the level surface $\beta_{\lambda}=h^{-1}(\lambda)$ and $D(u ; \lambda)$ for the Dirichlet integral over the region $\Omega_{\lambda}$ bounded by $\alpha$ and $\beta_{\lambda}$. The main result of this paper is the inequality: $\max h \mid \Omega_{\lambda}=m(h ; \lambda)=D(h ; \lambda)<m(u ; \lambda)<D(u ; \lambda)$, for $u \in H_{0}(\Omega), u \neq \pm h$. Thus $h$ minimizes the mean and the Dirichlet integral in the class $H_{0}(\Omega)$. This inequality can be used in the classification of locally Euclidean spaces. In particular, we shall show that $O_{G}=O_{H_{0} M}=O_{H_{0} D}=O_{H_{0} B}$. Here $O_{G}$ is the class of spaces $V$ possessing no Green's function, and $O_{H_{0} K}, K=M, D, B$ is the class of $V$ on which there exist no nonconstant $H_{0} K$ functions, that is, harmonic functions which vanish on the border of a boundary neighborhood of $V$ and which are of finite mean, of finite Dirichlet integral, or bounded, respectively.

The methods employed here appear to be valid for arbitrary Riemannian $n$-spaces [1]. However, we shall assume that $V$ is a locally Euclidean (flat) $n$-space [2].

Nevanlinna [3] proved the above inequality in two dimensions by the use of complex variables. It was later used by Sario [4] in classifying Riemann surfaces.

The proof of the inequality will be carried out by means of two lemmas and their corollaries.

Lemma 1. Let $f$ be continuous on $\bar{\Omega}_{\lambda}$, the region bounded by $\alpha$ and $\beta_{\lambda}$. Then

$$
\frac{d}{d \lambda} \int_{\Omega \lambda} f d V=\int_{\beta \lambda} \frac{f}{|\nabla h|} d S .
$$

Received by the editors November 16, 1964 and, in revised form, February 1, 1968.

1 This paper is a revision of part of a Ph.D. dissertation written at University of California, Los Angeles, under the direction of Professor Leo Sario. 
Proof. We express the volume integral as an iterated integral $\int f d V=\iint f d S d t$, where $t$ is measured normal to the level surfaces of $h$. To express this last integral in terms of $h$, we use $(d h / d t)=|\nabla h|$, or $d t=(d h /|\nabla h|)$, so that

$$
\int_{\Omega_{\lambda}} f d V=\int_{0}^{\lambda} \int_{\beta_{\lambda}} \frac{f}{|\nabla h|} d S d h .
$$

This iterated integral is now differentiated with respect to $\lambda$. The zeros of $|\nabla h|$ do not affect the integral on the right because they form a set of zero capacity (cf. Kellogg [5, p. 273]).

Corollary 1. Let $u$ be a function in $H_{0}(\Omega)$. Then

$$
\frac{1}{2} \frac{d}{d \lambda}\left[m^{2}(u ; \lambda)\right]=\int_{\beta_{\lambda}} u \frac{\partial u}{\partial n} d S=D(u ; \lambda) .
$$

Proof. We first transform the integral for the mean to a volume integral, $m^{2}(u ; \lambda)=\int u^{2}(\partial h / \partial n) d S=\int u^{2} \nabla h \cdot n d S=\int 2 u \nabla u \cdot \nabla h d V$. Then by Lemma 1 ,

$$
\frac{d}{d \lambda}\left[m^{2}(u ; \lambda)\right]=2 \int_{\beta \lambda} u \nabla u \cdot \frac{\nabla h}{|\nabla h|} d S=2 \int_{\beta \lambda} u \frac{\partial u}{\partial n} d S .
$$

Corollary 2. If $u \in H_{0}(\Omega)$, but $u \neq \pm h$, then

$$
D^{\prime}(u ; \lambda)>\left(\int_{\beta \lambda}\left|\frac{\partial u}{\partial n}\right| d S\right)^{2} .
$$

Proof. We apply formula (1) to the Dirichlet integral:

$$
\begin{aligned}
D^{\prime}(u ; \lambda) & =\frac{d}{d \lambda} \int_{\Omega_{\lambda}}|\nabla u|^{2} d V=\int_{\beta \lambda} \frac{|\nabla u|^{2}}{|\nabla h|} d S \\
& =\int_{\beta \lambda}\left(\frac{|\nabla u|}{|\nabla h|}\right)^{2}|\nabla h| d S .
\end{aligned}
$$

We then use the Schwarz inequality: $\left(\int f g|\nabla h| d S\right)^{2} \leqq \int f^{2}|\nabla h| d S$ $\cdot \int g^{2}|\nabla h| d S$, and obtain

$$
D^{\prime}(u ; \lambda) \leqq\left(\int_{\beta \lambda} \frac{|\nabla u|}{|\nabla h|}|\nabla h| d S\right)^{2} \leqq\left(\int_{\beta \lambda}\left|\frac{\partial u}{\partial n}\right| d S\right)^{2} .
$$

Equality holds if and only if $|\nabla u| /|\nabla h|=t(\lambda)$ and $|\nabla u|=|\partial u / \partial n|$, which imply that $u= \pm h$.

Corollary 3. If $u \in H_{0}(\Omega)$ and $u \neq \pm$, then 


$$
m^{\prime}(u ; 0)>1 \text {. }
$$

Proof. On differentiating (2), we obtain $D^{\prime}(u ; \lambda)=\left[m^{\prime}(u ; \lambda)\right]^{2}$ $+m(u ; \lambda) m^{\prime \prime}(u ; \lambda)$. For $\lambda=0$, this reduces to $D^{\prime}(u ; 0)=\left[m^{\prime}(u ; 0)\right]^{2}$. Equation (3) with $\lambda=0$ implies that $\left[m^{\prime}(u ; 0)\right]^{2}=D^{\prime}(u ; 0)>1$. Taking the square root of this yields $(5)$ since $m(u ; 0)=0$ and $m(u ; \lambda) \geqq 0$ so that $m^{\prime}(u ; 0) \geqq 0$.

Lemma 2. If $u \neq \pm h$ is a function in $H_{0}(\Omega)$, then

$$
m^{\prime \prime}(u ; \lambda)>0 \text {. }
$$

Proof. We apply the above Schwarz inequality with $f=u$, $g=(1 /|\nabla h|)(\partial u / \partial n)$, to (2) and obtain

$$
\begin{aligned}
m^{2}(u ; \lambda) m^{\prime 2}(u ; \lambda) & =\left(\int_{\beta \lambda} u \frac{1}{|\nabla h|} \frac{\partial u}{\partial n}|\nabla h| d S\right)^{2} \\
& \leqq \int_{\beta \lambda} u^{2}|\nabla h| d S \int_{\beta \lambda} \frac{1}{|\nabla h|^{2}}\left(\frac{\partial u}{\partial n}\right)^{2}|\nabla h| d S \\
& \leqq m^{2}(u ; \lambda) \int_{\beta \lambda} \frac{|\nabla u|^{2}}{|\nabla h|} d S \leqq m^{2}(u ; \lambda) D^{\prime}(u ; \lambda)
\end{aligned}
$$

However, from $(2), D^{\prime}(u ; \lambda)=m(u ; \lambda) m^{\prime \prime}(u ; \lambda)+m^{\prime 2}(u ; \lambda)$. Substituting this in the above inequality and simplifying, we get $0 \leqq m^{3}(u ; \lambda) m^{\prime \prime}(u ; \lambda)$. As before equality holds if and only if $u= \pm h$.

We can now prove the main inequality. From (5) and (6), we see that

$$
m^{\prime}(u ; \lambda)=\int_{0}^{\lambda} m^{\prime \prime}(u ; \lambda) d \lambda+m^{\prime}(u ; 0)>m^{\prime}(u ; 0)>1 .
$$

This together with equation (2) yields $D(u ; \lambda)>m(u ; \lambda)$. Since $\int(\partial h / \partial n) d S=1$ on $\beta_{\lambda}, D(h ; \lambda)=m(h ; \lambda)=\lambda$. Thus we have the desired result:

$$
\begin{aligned}
D(u ; \lambda) & >m(u ; \lambda)=\int_{0}^{\lambda} m^{\prime}(u ; \lambda) d \lambda \\
& >\int_{0}^{\lambda} d \lambda=\lambda=D(h ; \lambda)=m(h ; \lambda) .
\end{aligned}
$$

We shall now use this inequality to prove that the classes of Euclidean spaces defined in the introduction to this paper coincide.

TheOREM. For locally Euclidean $n$-spaces,

$$
O_{G}=O_{H_{0} K}, \quad K=B, M \text {, or } D .
$$


Proof. Let $W$ be a regular subregion of $V$ with border $\alpha$. We consider an arbitrary regular region $W^{\prime}$, with border $\beta$, which contains $W$. We set $\Omega=W^{\prime}-W$ and use the notation and terminology previously introduced. If $V \notin O_{H_{0} B}$, then there is a $W$ and $u$ such that $|u| \leqq K$ on $V-W$. We then have

$$
m^{2}(u ; k)=\int_{\beta} u^{2} \frac{\partial h}{\partial n} d S \leqq K^{2} \int_{\beta} \frac{\partial h}{\partial n} d S=K^{2} .
$$

Thus $m(u ; k)$ is uniformily bounded for every such $W^{\prime}$ and $V \notin O_{H_{0} M}$.

To prove the opposite inclusion, let $u \in H_{0} M(V-W)$. Then for $\Omega=W^{\prime}-W, m(u ; k) \leqq M$. The maximum principal together with the inequality (7) imply that $|h(z)| \leqq k \leqq M$. These harmonic functions are therefore uniformily bounded and there exists a sequence of them which converges to a harmonic function $h_{0}$ on $V-W$. Since each $|h(z)| \leqq M,\left|h_{0}(z)\right| \leqq M$ and $h_{0} \in H_{0} B(V-W)$. Inequality (7) also shows that $h_{0} \in H_{0} D(V-W)$.

That $H_{0} D(V-W) \subset H_{0} M(V-W)$ is an immediate consequence of (7) since the Dirichlet integral is a bound for the mean.

The harmonic measure $\omega_{\Omega}$ of $\beta$ with respect to $\Omega$ is the harmonic function with $\omega_{\Omega} \mid \alpha=0$ and $\omega_{\Omega} \mid \beta=1$. The harmonic measure of the ideal boundary of $V$ is, by definition, $\omega=\lim _{W^{\prime} \rightarrow V} \omega_{\Omega}$.

We shall finish the proof of the theorem by showing that $O_{G}$ $=O_{H_{0} B}$. If $V \notin O_{G}$ then $\omega \in H_{0} B(V-W)[6]$ and $V \notin O_{H_{0} B}$. If $V \notin O_{H_{0} B}$, let $|u| \leqq K$ on $V-W$. For every $\Omega=W^{\prime}-W, m(u ; k) \leqq K$. The harmonic measure $\omega_{\Omega}$ is related to $h_{\Omega}$ by $\omega_{\Omega}=h_{\Omega} / k_{\Omega}$. Since $k_{\Omega} \leqq K, \lim k_{\Omega}$ $\leqq K$. It follows that $\omega=\lim \left(h_{\Omega} / k_{\Omega}\right) \neq 0$ and therefore $V \notin O_{G}$ [6].

REMARK. This theorem provides a partial solution to problem (1), page 109 of Sario [7].

\section{REFERENCES}

1. S. Smith, Classification of Riemannian spaces, Doctoral dissertation, Univ. of Calif., Los Angeles, 1965, 57 pp. 1949.

2. L. P. Eisenhart, Riemannian geometry, Princeton Univ. Press, Princeton, N. J.,

3. R. Nevanlinna, Über Mittelwerte von Potentialfunktionen, Ann. Acad. Sci. Fenn. Ser. A.I. 57 (1949), 12 pp.

4. L. Sario, Sur la classification des surfaces de Riemann, Den 11 te Skandinaviske Matematikerkongress, Trondheim, 1949, pp. 229-238.

5. O. D. Kellogg, Foundations of potential theory, Dover, New York, 1953.

6. L. Ahlfors and L. Sario, Riemann surfaces, Princeton Univ. Press, Princeton, N. J., 1960.

7. L. Sario, Classification of locally euclidean spaces, Nagoya Math. J. 25 (1965), 87-111.

\section{BRIGHAM Young UNIVERSITY}

